\title{
Mechanical Ventilation and the Kidney
}

\author{
Jay L. Koyner ${ }^{a}$ Patrick T. Murray ${ }^{b}$ \\ a University of Chicago, Chicago, III., USA; ${ }^{b}$ University College Dublin School of Medicine and Medical Science, \\ Dublin, Ireland
}

\section{Key Words}

Acute kidney injury · Critical care nephrology $\cdot$ Acute lung injury $\cdot$ Mechanical ventilation $\cdot$ Cytokines

\begin{abstract}
Acute lung injury (ALI) and acute kidney injury (AKI) are complications often encountered in the setting of critical illness. Both forms of end-organ injury commonly occur in similar settings of systemic inflammatory response syndrome, shock, and evolving multiple organ dysfunction. Recent elucidation of the pathobiology of critical illness has led to a more basic mechanistic understanding of the complex interplay between injured organs in patients with multiple organ dysfunction syndrome; this has been aptly called 'the slippery slope of critical illness' [Kidney Int Suppl 1998;66:S25S33]. Distant organ effects of apparently isolated injuries to the lungs, gut, and kidneys have all been discovered in recent years. In this article, we will review the harmful bidirectional interaction between ALI and AKI, which appears to be a common clinical syndrome with routine clinical implications. We will review the current understanding of lung-kidney interactions from both perspectives, including the renal effects of ALI and mechanical ventilation, and the pulmonary sequelae of AKI. In this review of the emerging evidence of deleterious bidirectional organ cross talk between lung and kidney, we will focus on the role of ventilator-induced kidney injury in the pathogenesis of AKI in patients with ALI.

Copyright $\odot 2009$ S. Karger AG, Base
\end{abstract}

\section{Diagnosis - Definitions}

\section{Acute Kidney Injury}

Acute kidney injury (AKI), also called acute renal failure, is traditionally defined as the abrupt loss of kidney function with the concomitant retention of nitrogenous waste products such as urea, as well as abnormal regulation of fluid and electrolyte homeostasis. For decades, this decrease in kidney function has been detected through the elevation of blood creatinine concentrations from pre-insult levels. One of the greatest impediments to progress in the field of AKI has been the general lack of a standardized, consensus-derived creatinine-based definition. The lack of a consensus and uniformity makes the comparison of trials employing fundamentally different definitions of AKI difficult. Additionally, serum creatinine serves as a marker of glomerular filtration, and is not optimally suited to the detection and quantification of renal tubular injury per se. This disconnect, the employment of a marker of glomerular filtration for quantifying tubular injury, has served to further hamper progress in the clinical study of AKI. As discussed elsewhere in this special edition of Blood Purification, recent attempts have been made to revamp and standardize these definitions of AKI $[1,2]$. The use of the Acute Kidney Injury Network (AKIN) and Risk, Injury, Failure, Loss, End Stage (RIFLE) Criteria has led to some progress in the clinical study of AKI and these classification schemes are described in previous articles in this journal.

\section{KARGER}

Fax +4161306 1234 E-Mail karger@karger.ch www.karger.com (c) 2009 S. Karger AG, Basel

0253-5068/10/0291-0052\$26.00/0

Accessible online at:

www.karger.com/bpu
Patrick T. Murray, MD

UCD School of Medicine and Medical Science, Catherine McAuley Centre

Mater Misericordiae Hospital

Nelson Street, Dublin 7 (Ireland)

Tel. +353 1716 6319, Fax +3531716 6355, E-Mail patrick.murray@ucd.ie 
The recent discovery of a variety of novel urinary biomarkers of AKI (including neutrophil gelatinase-associated lipocalin, NGAL, kidney injury molecule-1, interleukin-18, cystatin C), which can detect AKI earlier than serum creatinine, has generated interest in the potential revision of the definition to include these biomarkers, similar to the way that troponin and other cardiac biomarkers aid in the definition of acute coronary syndromes [3-6]. The clinical utilization of these biomarkers is not yet a reality, but their discovery and clinical validation can be expected to improve the definition and study of human AKI.

\section{Mechanical Ventilation/Acute Lung Injury}

A mechanistic classification system for patients requiring mechanical ventilation (MV) has been devised based upon the four pathophysiologic patterns of respiratory failure [7]. Type I respiratory failure (acute hypoxemia) results from a failure of oxygenation due to increased intrapulmonary shunting of blood [characterized by an increased fraction of cardiac output passing through the pulmonary circulation without reoxygenation, referred to as $\left.\uparrow \mathrm{Q}_{\text {shunt }} / \mathrm{Q}_{\mathrm{t}(\text { cardiac output })}\right]$, and is classically seen in the setting of pulmonary edema (both cardiogenic and noncardiogenic acute lung injury, ALI/acute respiratory distress syndrome, ARDS) as well as pneumonia, ventilation-perfusion mismatch and pulmonary hemorrhage. Type II respiratory failure (hypoventilatory) occurs in the setting of the failure of alveolar ventilation leading to the retention of carbon dioxide (hypercapnia). This hypercapnia is associated with reduced partial pressure of oxygen $\left(\mathrm{PaO}_{2}\right)$ and will generally correct with supplemental oxygen therapy. Type II respiratory failure is classically associated with decreased central nervous system drive (e.g. sedative drug overdose), respiratory muscle weakness (generally caused by neuromuscular diseases such as myasthenia gravis, amyotrophic lateral sclerosis (ALS), etc.), or increased respiratory system load leading to fatigue (increased pulmonary deadspace and/ or increased work of breathing (chronic obstructive pulmonary disease, asthma). Type III respiratory failure ('perioperative') relates to the susceptibility of the pulmonary system to atelectasis in the perioperative period. Type III respiratory failure is associated with decreased end-expiratory lung volumes and the progressive collapse of dependent alveolar units. Type III respiratory failure is exacerbated by patient's physical characteristics (supine position, obesity, abdominal wall stiffness, etc.). Finally, patients with type IV respiratory failure (shock) fall outside the specific definitions of the first three cat- egories, and require MV for support in the setting of systemic hypoperfusion (cardiogenic, hypovolemic, septic, or other forms of shock) associated respiratory muscle fatigue and increased minute ventilation requirements (systemic inflammation, hypermetabolism, metabolic acidosis). Of course, individuals with type IV respiratory failure may share many of the primary features of types I, II or III. The pathophysiologic features that underlie this classification scheme provide the basis for the selection and titration of MV modalities for these patients, and we further discuss how these classifications relate to specific clinical scenarios throughout this review.

ALI is a clinical syndrome characterized by acute (less than 7 days) onset of severe hypoxemia and bilateral pulmonary infiltrates in the absence of elevated left atrial pressures [8]. Alternatively, ALI has been defined as a subject who has a ratio of partial pressure of oxygen in the arterial blood $\left(\mathrm{PaO}_{2}\right)$ to the inspired fraction of oxygen $\left(\mathrm{FiO}_{2}\right)$ of less than 300 . ARDS is the most severe form of ALI where this ratio $\left(\mathrm{PaO}_{2} / \mathrm{FiO}_{2}\right)$ is less than 200 [9]. ALI and ARDS occur in the setting of a variety of clinical scenarios including sepsis, trauma, blood product transfusion, pancreatitis, burns as well as pneumonia and other forms of lung injury (inhalation, aspiration etc.).

It is these concrete definitions of ALI and its severity that have led to the successful identification of patients for several large multicenter clinical trials in ALI $[10,11]$. The results of these trials will be discussed in the review below, but it deserves noting that while these trials are not always positive, their protocols have led to standardization in the care of patients with ALI. This is in stark contrast to the AKI field, in which clinical investigation has been less successful.

When AKI has been investigated in the setting of ALI, the crude definitions of AKI employed did not reflect the increasing use of the standardized RIFLE or AKIN criteria, or any prior system. In the seminal ARDSNet tidal volume trial [10], the investigators defined AKI (renal failure) as a serum creatinine over $2.0 \mathrm{mg} / \mathrm{dl}(177 \mu \mathrm{M})$, with no reference to the subjects baseline value. Despite this rudimentary definition, they were still able to demonstrate that those treated with a lung-protective lower tidal volume incurred statistically fewer days with AKI compared to those exposed to the lung-injurious ventilation strategy (mean $\pm \mathrm{SD}, 18 \pm 11$ vs. $20 \pm 11$ days, $\mathrm{p}=$ $0.005)$ [10]. This same AKI definition $(>2.0 \mathrm{mg} / \mathrm{dl})$ was employed in the comparison of a fluid liberal and fluid conservative strategy in ALI (FACTT trial); here, however, the investigators did not find a difference in AKI rates within the first 7 days on the protocol $(p=0.45)$, or 
the first 28 days $(p=0.59)$ [11]. Similarly, there was no significant difference between the need for renal replacement therapy (RRT) between these two groups; with $10 \%$ of the fluid-conservative arm and $14 \%$ of the fluid-liberal arm $(p=0.06)$ requiring RRT. While AKI and RRT rates were prespecified secondary endpoints in both of these trials, we further discuss the primary endpoint as well as the interpretation of this RRT data later in this review and would urge that subsequent ALI/ARDSNet trials utilize more sensitive and standardized AKI definitions and staging criteria.

Finally, there is a growing body of literature demonstrating that not only are there novel plasma and urinary biomarkers that can diagnose AKI earlier than plasma creatinine, but these same markers may be able to play a role in predicting patient outcomes in ALI/ARDS. Utilizing samples from the original ARDSNet trial, Parikh et al. [12] demonstrated that urinary IL-18 levels were elevated earlier than plasma creatinine, and that urinary IL18 values were also significantly different between survivors and nonsurvivors $(\mathrm{p}<0.05)$. Furthermore, on multivariable analysis, the urine IL-18 concentration on day 0 is an independent predictor of mortality. Similarly, Liu and colleagues tested plasma samples from over 850 subjects from the first ARDSNet trial $[10,13,14]$ to investigate novel plasma biomarkers of AKI. AKI was defined as a $50 \%$ increase in creatinine within the first 4 days of study enrollment. They demonstrated increased AKI rates with this more standardized definition $(n=209$, $24 \%$ of those included in their substudy) and found that mortality was $58 \%$ in those with AKI compared to $28 \%$ in those without AKI $(\mathrm{p}<0.001)$. They measured the association of baseline levels of a variety of biomarkers (including IL-6, IL-8, IL-10, soluble tumor necrosis factor receptor- $\alpha$ type I and II (STNFR I and II), plasminogen activator inhibitor 1 (PAI-1), and intracellular adhesion molecule-1). Through a multivariable model and adjustments for severity of illness, interventions and baseline demographics, they found that IL- $6(\mathrm{p}=0.04)$, PAI-1 $(\mathrm{p}<0.01)$ and STNFR I $(\mathrm{p}=0.001)$ and II $(\mathrm{p}=0.02)$ levels were all independently associated with AKI. Finally, they constructed a predictive model which demonstrated that a combination of clinical predictors and biomarkers outperformed (via ROC curves) each parameter individually; with PAI-1 and TNF- $\alpha$ type I having highly significant contributions to the model [15]. IL-6, PAI-1 and STNFR I and II were all independently associated with the development of AKI and all apparently play an important role in patient outcomes in the setting of ALI [15].

\section{Epidemiology}

\section{Acute Lung Injury/Acute Respiratory Distress Syndrome}

While the widespread use of concrete definitions has lead to progress in the treatment of ALI and ARDS, the worldwide incidence of ALI remains unclear. In 2005, Rubenfeld et al. [16] reported a prospective, populationbased, cohort study in 21 hospitals in and around King County, Wash., USA, from April 1999 through July 2000, using a validated screening protocol to identify patients who met the consensus criteria for ALI/ARDS. Based on the 1,113 patients who met the criteria for ALI, the crude incidence was 78.9 per 100,000 person-years, with the age-adjusted incidence being 86.2. Note that they demonstrated that both the incidence as well as mortality of ALI and ARDS increased with advancing age. Similar to previous data, they reported a mortality rate of $38.5 \%$ [16].

While data regarding the incidence of ALI have varied, mortality rates have generally ranged from 25 to $61 \%$ $[10,11,17-19]$. The variation in incidence is likely multifactorial. For example, in a prospective multinational cohort of 78 European intensive care units (ICUs), BrunBuisson et al. [18] described ALI in 7.1\% of all 6,522 screened admissions. This study, entitled The Acute Lung Injury Verification of Epidemiology (ALIVE), demonstrated that over $54 \%$ of those who present with ALI went on to develop ARDS. Unfortunately, this study only described admission during a discrete 2-month study period and did not allow for a seasonal variation in ALI/ ARDS rates. Similarly, Bersten et al. [17] set out to determine the incidence and mortality of ALI/ARDS in a 2month prospective cohort study of three Australian States between October and November 1999. They found the incidence of ALI was 34 cases per 100,000 - with ARDS occurring in over $88 \%$ of cases of ALI (148 of 168 cases). Separately, in an audit of all ICU admissions for 23 Scottish ICUs, Hughes et al. [19] demonstrated a rather low incidence of ARDS; 16 cases per 100,000 person-years. They also had a rather high mortality rate of $60.9 \%$. The variation in the observed incidence of ALI/ARDS is likely multifactorial and includes varying exposure to ALI risk factors, genetics, race, different underlying causes of ALI, and access to health care [20,21]. Finally, as we will discuss in detail in this review, the deleterious effects of harmful modes of mechanical ventilation on injured lungs and distant organs (including the kidneys) may in part explain the outcome differences in the aforementioned studies. 


\section{Pathophysiology}

\section{Renal Effects of ALI and Mechanical Ventilation}

$\mathrm{AKI}$ is an independent predictor of mortality in ICU patients [22]. Unfortunately, AKI often develops as a component of multi-organ system dysfunction in critically ill patients and may lead to mortality rates in excess of $60 \%$ [22-25]. Severe AKI in critically ill patients is typically part of a triad with shock and respiratory failure requiring positive-pressure mechanicalventilation (PPV) [26]. The physiologic impact of PPV and its effects on renal perfusion and function are well documented [27]. Recent advances in critical care, including the implementation of lung-protective ventilatory strategies, have disclosed the role of inflammatory mediators of ALI and specifically ventilator-induced lung injury in the pathogenesis of AKI, which in some cases might appropriately be called ventilator-induced kidney injury (VIKI).

\section{Effects of Mechanical Ventilation and ALI on Renal} Function

\section{Hemodynamic Effects of PPV}

In 1947, PPV was first shown to affect renal function and perfusion [27]. Since this first study involving healthy individuals receiving continuous positive airway pressure, a variety of mechanisms have been shown to alter cardiac output and result in changes of renal perfusion and function [28]. While a variety of animal and human studies have produced equivocal results, there are believed to be two main components contributing to the decrease in renal perfusion and function caused by PPV, broadly categorized as hemodynamic and neurohormonal [29-32]. Increased intrathoracic pressure associated with PPV decreases the venous return to the heart (preload) and may result in decreased cardiac output [30]. This may lead to hypotension and fluid-responsive shock, which is common in the initial postintubation period when PPV is initiated. The increase in intrathoracic pressure has been shown to correlate with a decrease in renal plasma flow, glomerular filtration rate (GFR) and urine output during PPV [29]. This aspect of renal hemodynamics has in part been validated by the canine work performed by Qvist et al. [33], who showed that a stable cardiac output in the setting of PPV is not associated with a decrease in GFR or urine output. There are other, clinically occult adverse hemodynamic effects of PPV in the pulmonary, systemic, and renal circulations. PPV has been shown to compress the mediastinal structures and pulmonary vasculature and may result in increased right ventricular afterload. This may result in a decreased car- diac output and a decline in renal perfusion independent of effects on venous return. Similarly, PPV in patients with increased intrathoracic pressure (injured, stiff lungs or chest wall) or intra-abdominal pressure (morbid obesity, abdominal compartment syndrome) may act to decrease renal blood flow by increasing renal venous pressure (which diminishes renal perfusion pressure) and by compressing the renal vasculature, thus leading to AKI [34-36]. However, because effects on cardiac output and renal perfusion are insufficient to fully explain the mechanism of PPV-induced oliguria and renal dysfunction, other mechanisms have been proposed to explain these phenomena.

\section{Neurohormonal Effects of PPV}

PPV has been shown to alter a variety of neurohormonal systems including sympathetic outflow, the reninangiotensin axis, nonosmotic vasopressin $(\mathrm{ADH})$ release and atrial natriuretic peptide (ANP) production. The end result of all of these neurohormonal pathways is diminished renal blood flow, decreased GFR, and fluid retention (salt and water) with oliguria. Despite conflicting data, there is some evidence that fluid retention is caused by PPV-induced production of vasoactive substances. These mediators shift intrarenal blood flow from the cortex to the medulla, resulting in greater fluid retention at any level of renal perfusion $[30,37,38]$.

$\mathrm{ADH}$ release in the setting of PPV is likely multifactorial. While some evidence suggests that decreased atrial stretch due to the absolute or relative intravascular volume depletion associated with mechanical ventilation plays a role in the increased ADH secretion [39], it is clear that there are more factors at play [40]. Several experimental denervation procedures (cervical, carotid sinus) in animals have blunted the $\mathrm{ADH}$ response, but none has been able to abolish it $[40,41]$. Taken together with evidence that an increase in urine osmolarity is not commonly found as a direct effect of PPV, this suggests that $\mathrm{ADH}$ release is not the primary mechanism responsible for the decrease in urine output during PPV.

It should come as no surprise that the renin-angiotensin-aldosterone axis is affected by PPV. PPV has been shown to increase plasma renin activity in both animal models and humans [29, 41, 42]. Animal denervation studies have shown that increased sympathetic flow indirectly leads to the increase in renin activity [40]. Thus, PPV leads to increased sympathetic tone, secondary activation of the renin-angiotensin axis, and decreased renal plasma flow, GFR and urine output. Of course, downstream stimulation of aldosterone production is among 
the salt-retaining effects of PPV. The limited studies investigating catecholamine levels and PPV have not provided a clear correlation between mechanical ventilation and hormone concentrations [43].

Suppression of ANP release has been proposed as a source of the decreased urine volume and sodium content associated with PPV. Using a canine model, Ramamoorthy et al. [44] showed that plasma ANP levels correlated with right atrial filling pressures and decreased with the initiation of PPV ( $p<0.05)$. While some animal and human studies have supported these data, other human studies have refuted them $[42,45]$. Of course, even if ANP suppression does play a role in causing PPV-induced sodium retention and oliguria, it may not explain the reported decrease in GFR caused by PPV.

\section{Pathophysiologic Effects of AKI on Pulmonary}

Function

In spite of the high mortality rate associated with concomitant AKI and ALI and their clear clinical link, few studies have examined the role of the kidney in the pathogenesis and development of ALI. There are both experimental animal data and limited human study results that describe effects of AKI and the use of RRT on pulmonary function, including the development of ALI as a consequence of AKI, and the effects of RRT on the management and outcomes of patients with ALI combined with renal failure.

\section{Pulmonary Effects of AKI}

The link between AKI and the lungs dates back to the 1950s when it was first hypothesized that the patients with AKI had abnormal chest X-rays [46]. AKI was thought to lead to increased pulmonary vascular permeability and pulmonary congestion; thus, the term 'uremic lung' was coined [47]. Since that time, AKI patients have experienced the benefits of RRT and while the term 'uremic lung' had fallen by the wayside, recent advances in understanding the complex interplay between AKI and the pulmonary system have led to a rejuvenation of this term [46].

Many of the effects of AKI are difficult to identify and quantify independently, but experimental data are increasingly elucidating the subclinical effects of AKI on distant organ function [48]. It is obvious that problems such as refractory hyperkalemia, pulmonary edema, or uremic manifestations such as pericarditis are related to AKI when they acutely develop in the appropriate setting. Other uremic manifestations may have several explanations (encephalopathy, acidosis), or may be occult causes of other complications (bleeding diathesis and gastrointestinal bleed, leukocyte dysfunction with immunosuppression and nosocomial infection). In addition to the emerging data that appear to confirm an independent role of AKI in increasing mortality in the ICU, it is also clinically obvious that AKI is a cause of significant morbidity (including ALI and other distant organ injury) and severely complicates ICU management [49].

\section{Basic Science}

Inflammatory Mediators and Ventilator-Induced Lung and Kidney Injury

Mounting evidence points to the role of cytokines and chemokines in the pathogenesis of ARDS [21, 50,51]. Emerging data further suggest that the pro-inflammatory effects of PPV may be a source of AKI, especially in the setting of mechanical ventilation and lung-injurious ventilator strategies (higher tidal volumes and lower positive end-expiratory pressure, PEEP) [10, 52-54]. The pathogenesis of VIKI was elucidated in part by Douillet et al. [55], who showed that mechanical ventilation itself can alter nucleotide and purinoceptor expression in the kidney. They used a four-way rodent model (altering tidal volume and PEEP, as well as the use of a placebo) to show that expression of these stress-responsive molecules is altered even in the presence of lung-protective mechanical ventilation strategies. Since these extracellular ligands, which help control vascular tone and epithelial/ endothelial permeability, are altered by ventilatory strategy, others have looked at the cellular and molecular effects of similar measures. Imai and colleagues previously employed a rabbit model of ALI (acid aspiration) with both injurious and noninjurious ventilator strategies and variable oxygen and carbon dioxide levels to elegantly demonstrate that injurious ventilator strategies precipitate AKI. They demonstrated that injurious mechanical ventilation strategies induced production of a variety of inflammatory cytokines (IL-8 and monocyte chemotactic protein-1, amongst others). They further demonstrated that this injurious strategy induced epithelial cell apoptosis in both the kidneys and intestines, providing concrete evidence of injurious distant organ cross talk initiated by PPV-requiring ALI leading to VIKI. They further proved the mechanistic hypothesis underlying VIKI by showing that plasma from injuriously ventilated rabbits induced apoptosis in fresh, healthy rabbit proximal tubular cells, to a significantly greater extent than 
plasma from controls that received lung-protective ventilation [52].

Employing a similar animal model of ALI, Kuiper et al. [56] demonstrated no significant difference in plasma IL-6 levels in rats that were exposed to lung-injurious (peak inspiratory pressure of $20 \mathrm{~cm}$ of $\mathrm{H}_{2} \mathrm{O}$ with a PEEP of $2 \mathrm{~cm} \mathrm{H}_{2} \mathrm{O}$ ) compared to those using a lung-protective ventilatory strategy (peak inspiratory pressure of $14 \mathrm{~cm}$ of $\mathrm{H}_{2} \mathrm{O}$ with a PEEP of $5 \mathrm{~cm} \mathrm{H}_{2} \mathrm{O}$ ) and nonventilated rats. This study found a $40 \%$ reduction in the renal blood flow in the lung-injurious rodents, but this decreased perfusion was not associated with any significant effect on renal function or histology. Additionally, they demonstrated that lung-injurious ventilation led to a significant increase in renal endothelin-1 production, presumably leading to increased renal vasoconstriction [56].

More recently, Hoag et al. [57] found interesting results in a canine model of acid aspiration, which provide an additional mechanistic insight. Utilizing 11 animals ( $\mathrm{n}=6$ for ALI and $\mathrm{n}=5$ for control), they induced lung injury while attempting to maintain identical hemodynamic parameters and arterial blood gas values $\left(\mathrm{pCO}_{2}\right.$ and $\mathrm{HCO}_{3}$ ) across the two groups. They demonstrated no difference in renal function, as measured by renal blood flow, urine flow, serum creatinine and renal histology scores. Similarly, there was no difference in serum cytokine levels (TNF- $\alpha$, IL-10, IL-6 and interferon- $\gamma$ ), although these measurements (cytokines and renal blood flow) were only made in 2 animals from each group. Further investigation of the effects of experimental ALI in the setting of stable hemodynamic parameters is warranted.

\section{Role of Nitric Oxide in Animal Models of ALI}

Nitric oxide, a vasodilator whose metabolites have been shown to exert systemic and renal cytotoxic effects via oxidative stress mediators, has been shown to play key roles in a variety of cellular functions, including vascular and epithelial permeability and apoptosis [58]. It is also well documented that nonselective inhibition of nitric oxide synthase (NOS) leads to elevated systemic blood pressure and marked renal vasoconstriction. Using a rodent model of ventilator-induced lung injury, Choi et al. [59] showed that PPV with injurious high tidal volumes $(20 \mathrm{ml} / \mathrm{kg})$ induced NOS expression in both the lung and the kidney. At the same time, an increase in systemic microvascular leak was also seen in both organs. This robust detrimental NOS-mediated response to injurious ventilation was blunted when the animals undergoing PPV were adequately fluid resuscitated, and the improvement in renal function was augmented using the NOS inhibitor (N-nitro-L-arginine methyl ester). In addition to changes in NOS and its metabolites, they showed that serum vascular endothelial growth factor (VEGF) levels were increased in those animals receiving the lung-injurious ventilation; it is known that nitric oxide increases vascular permeability via extracellular signal-regulated kinases 1 and 2 and VEGF [59]. Thus, it appears that increased vascular permeability and associated cytokine release are contributors to the development of AKI in the setting of PPV. In summary, the decreased morbidity and mortality of patients with ALI achieved with lung-protective strategies for mechanical ventilation are likely mediated not only by amelioration of ventilator-induced lung injury and inflammation, but also by diminished injurious cross talk with distant organs, including the kidneys.

Permissive Hypercapnia and Its Effects on Cytokines

A significant body of experimental literature suggests that hypercapnic acidosis has cytoprotective and anti-inflammatory effects. In isolated perfused animal hearts, hypercapnic acidosis (type II respiratory failure) has been shown to have a dose-dependent protective effect against myocardial ischemia-reperfusion injury [60]. Similar degrees of metabolic acidosis do not offer the same level of protection from ischemia-reperfusion injury as respiratory acidosis [60]. The protective effect of hypercapnic acidosis may result from the inactivation of calcium channels (leading to regional vasodilation), a reduction in cellular oxygen demand [61], or anti-inflammatory effects [62-64]. Hypercapnia can decrease the conversion rate of I- $\kappa \mathrm{B}$ to NF- $\kappa \mathrm{B}$, and accordingly decrease the release of cytokines (IL-8 and ICAM-1) that are implicated in the pathogenesis of ALI, AKI, and other forms of tissue injury [64]. Similarly, hypercapnia and associated intracellular acidosis decrease the release of other cytokines (TNF- $\alpha$ and IL-1) by inhibition of toll-like receptors [62, 63].

In contrast, O'Croinin et al. [65] recently conducted a randomized controlled animal study to investigate the effect of hypercapnia for $48 \mathrm{~h}$ on the severity of bacterial pneumonia. Using a rodent model of Escherichia coli pulmonary instillation, they demonstrated that hypercapnic rats (exposure to $\mathrm{FiCO}_{2}$ of $5 \%$ for $48 \mathrm{~h}$, compared to $0 \%$ ) had more acidosis, more extensive lung injury, increased cellular inflammation and increased bacterial colony counts compared to normocapnic-infected controls. Of note, both of these groups displayed significantly increased cellular inflammation and lung damage compared to both normocapnic and hypercapnic noninfected 
controls. Bronchoalveolar lavage (BAL) fluid was similar between the two infected groups including the total neutrophil count as well as IL-1 $\beta$ and macrophage inflammatory protein 2 . Interestingly, there was a difference in the percentage of neutrophils displaying active phagocytosis of fluorescently labeled latex beads, with the normocapnic-infected mice displaying increased phagocytosis compared to the hypercapnic-infected cohort $(\mathrm{p}<0.05)$ [65]. This work raises concerns that in the setting of ALI requiring mechanical ventilation, permissive hypercapnia might exacerbate the severity of intrapulmonary infections via impaired phagocytosis. More broadly, these data suggest that any beneficial effects of the use of decreased tidal volumes with attendant permissive hypercapnic acidosis may be offset by harmful effects on local antimicrobial defenses if ALI is the result of a primary pulmonary infection (including ventilator-associated pneumonia) rather than a noninfectious direct lung insult (e.g. acid aspiration) or systemic inflammatory syndrome (non-pulmonary sepsis, trauma, ischemia-reperfusion injury, etc.).

In contrast, Chonghaile et al. [66] have also used this rodent model in an attempt to describe the role that randomization to intravenous antibiotics (ceftriaxione 30 $\mathrm{mg} / \mathrm{kg}$ ) plays in a rodent model of E. coli-instilled pneumonia and hypercapnia $\left(\mathrm{FiCO}_{2}\right.$ of $\left.5 \%\right)$ compared to those experiencing a normocapnic environment. In the absence of antibiotics, hypercapnia reduced the bacterial pneumonia-induced increase in peak airway pressures and the decrease in the static lung compliance compared with control conditions (no antibiotics in a $0 \% \mathrm{FiCO}_{2}$ environment). In the presence of ceftriaxone, not only were bacterial counts decreased but hypercapnia attenuated the histologic pulmonary injury [66]. These data point to the potential improved ventilatory response/lung mechanics in the setting of hypercapnia, as well as the ability of appropriate antibiotics to attenuate the lung injury caused by pneumonia. Clearly, much work is still needed to reconcile the conflicting literature concerning the net effects of hypercapnia on neutrophil function, cytokine production, and cellular responses in ALI requiring mechanical ventilation.

\section{Animal Models of ALI and Distant Organ Effects}

Kramer et al. [67] have extensively investigated the mechanisms which link AKI and ALI. They were amongst the first to demonstrate this organ cross talk in a rat model of renal ischemia-reperfusion. They investigated lung vascular permeability (with Evan's blue dye extravasation) and the role of macrophages in pulmonary inflam- mation following $30 \mathrm{~min}$ of renal ischemia. Using CNI1493, a macrophage pacifant that inhibits the release of macrophage-derived inflammatory products, they demonstrated that $48 \mathrm{~h}$ after renal ischemia, animals pretreated with CNI-1493 had significantly reduced pulmonary vascular permeability and less lung injury compared to those receiving placebo/sham $(n=5$ per group, $\mathrm{p}<$ $0.05)$.

Since this original work, Rabb et al. [68] have generated a variety of rodent models investigating the distant organ cross talk of AKI. In a four-way rodent model (unilateral ischemic injury, bilateral ischemic injury, bilateral nephrectomy and sham/placebo) they demonstrated that ischemic AKI leads to alteration in pulmonary gene and protein expression. Specifically, bilateral ischemic AKI downregulates the pulmonary expression of all of the following: epithelial sodium channel (eNaC), $\mathrm{Na} / \mathrm{K}$-ATPase and aquaporin-5. It should be noted that unilateral ischemic injury did not lead to alteration of the pulmonary expression of these proteins, in contrast to bilateral renal ischemia-reperfusion or nephrectomy. Thus, the observed increase in lung permeability is likely mediated by a systemic effect of AKI, rather than a dose-dependent effect of the 'reperfusion products' of ischemic renal injury [68]. Subsequently, a variety of animal models have shown that pulmonary expression of these proteins (eNaC, $\mathrm{Na}, \mathrm{K}-\mathrm{ATP}$ ase and aquaporins) play important roles in the salt and water/fluid handling and permeability of alveolar epithelium; and dysregulation of these mechanisms may result in the development of ARDS in humans [69-71].

In mice, Hoke et al. [72] employed a similar experimental protocol (sham, unilateral renal ischemia, bilateral renal ischemia and bilateral nephrectomy) and demonstrated that AKI leads to altered pulmonary cytokine expression. Similar to the above studies, they showed that bilateral ischemic injury and bilateral nephrectomy were associated with statistically significant increases (compared to unilateral injury and sham) in several serum cytokine levels (IL-1 $\beta$, IL- 6 and IL-12). Of note, there were several other cytokines that increased in the setting of bilateral injury or bilateral nephrectomy, but not both, compared to the same controls (GM-CSF, IL-10 and keratinocyte-derived chemokines) [72]. Thus, both models for the acute loss of renal function displayed similar but uniquely different patterns of cytokine expression. Utilizing a similar but not identical protocol, Hassoun et al. [73] demonstrated that the global gene profiles from murine lungs exposed to ischemia-reperfusion AKI or bilateral nephrectomy differed quite significantly. Using the 
bilateral nephrectomy model, Hoke et al. [72] prophylactically administered the anti-inflammatory agent IL-10. They found that prophylaxis with IL-10 decreased the intensity of the inflammatory response induced by nephrectomy.

Klein et al. [74] subsequently used similar models (ischemic AKI, bilateral nephrectomies and sham procedures) in IL-6-deficient and wild-type mice. They demonstrated that lung injury is in part dependent on IL-6, which plays a role in neutrophil recruitment (as measured by macrophage inflammatory protein 2 and keratinocyte-derived chemokines, $\mathrm{KC}$ ) and pulmonary capillary leak (measured via lung pathology and Evans blue dye). Additionally, they administered an IL-6-inactivating antibody to the wild-type mice and displayed decreased evidence of lung injury (myeloperoxidase and KC levels) - pointing to a potential role for IL-6 inhibition to prevent lung injury in the setting of AKI.

More recently, Dodd-o et al. [75] induced ischemic AKI (or sham) in mice and observed the effect of a variety of postprocedure ventilatory strategies on lung injury. In assessing the differences between mice who were permitted to spontaneously ventilate and those receiving mechanical ventilation with low and high tidal volumes ( 7 or $30 \mathrm{ml} / \mathrm{kg}$ ), they controlled acid-base status by altering ventilatory dead space (preventing over ventilation), controlled volume status with appropriate fluid resuscitation, and selected appropriate fluids to minimize hyperchloremic metabolic acidosis. They found that animals with AKI and low tidal volume ventilation had increased pulmonary capillary leak (Evans blue dye) compared to controls without AKI. This increased leak was associated with decreased oxygenation but not with an increase in BAL fluid total protein or BAL neutrophils. In those mice exposed to high tidal volume (30 $\mathrm{ml} / \mathrm{kg}$ ) ventilatory strategy, AKI did not worsen the ALI as measured by the Evans blue dye, oxygenation, and BAL (protein and neutrophils). However, animals with AKI had fewer neutrophils in the BAL fluid compared to controls, which suggests that the AKI may lead to reduced airspace inflammation. The finding that AKI is associated with decreased pulmonary neutrophil recruitment in the setting of ALI is in agreement with the findings of Zarbock et al. [76]. Using a murine model of ALI (acid aspiration), Zarbock et al. [76] demonstrated that neutrophils are less efficiently recruited to the lung in setting of AKI, compared to nonuremic neutrophils. Taken together, these two studies suggest that effect of AKI on neutrophil migration may in fact decrease the deleterious effect of high tidal ventilation, but potential- ly worsen the severity of ALI in the setting of low tidal volume ventilation.

Finally, similar to the findings of Hoke et al. [72] using IL-10, other data suggest the potential utility of anti-inflammatory therapy to diminish ALI secondary to AKI. A rodent ischemia model was utilized to show that the administration of $\alpha$-melanocyte-stimulating hormone $(\alpha-\mathrm{MSH})$ immediately before reperfusion (following renal ischemic injury) reduced both kidney and lung injury. Additionally, this therapy prevented the activation of kidney and lung transcription factors, and decreased the expression of stress response genes (TNF- $\alpha$, and lung intracellular adhesion molecule-1) [77]. It is believed that $\alpha-\mathrm{MSH}$ acts by inhibition of the activation of transcription factors and stress response genes. This emerging data warrants further investigation of the use of IL-10 and other anti-inflammatory agents as potential therapeutic options for early AKI.

\section{Genomics and Animal Models of ALI/AKI}

Emerging data continue to underscore the complexity of kidney-lung interactions in AKI. Kim et al. [50] determined that ALI developing secondary to renal ischemiareperfusion in a rodent model is distinct from the ALI that develops in a rodent model of sepsis. While both the sepsis and ischemia models lead to increased pulmonary vascular permeability and pulmonary histology consistent with ARDS, they were distinct in that the ischemiareperfusion was associated with lower levels of pulmonary inflammatory cell infiltration, and each animal model had significantly different expression of a variety of heat shock proteins. Furthermore, pulmonary expression of a variety of cytokines (including TNF- $\alpha$ and proinflammatory chemokines) differed between animal models of lung injury, indicating that distinct inflammatory mediator profiles were involved in the different injury mechanisms [50].

More recently, Grigoryev et al. [78] have further described the local and systemic inflammatory transcriptome following AKI. Utilizing their previously described murine model of ischemia-reperfusion, they defined those genes whose renal and pulmonary tissue expression were significantly affected during both the early $(6 \mathrm{~h})$ and late $(36 \mathrm{~h})$ phases of AKI. Their functional genetic analysis identified several potential mediators of local and systemic inflammation (in both the lung and kidney), including NGAL (also known as lipocalin-2 LCN2; a novel urinary biomarker of AKI) which was the most upregulated gene in the kidney at both the early and late time points. Additionally, pulmonary expression of NGAL 
was increased at both time points, with more dramatic changes occurring at the 36-hour time point [78]. Finally, the phenomenon of distant organ injury resulting in ALI is not unique to renal ischemia, as gut ischemia has been shown to alter systemic cytokine levels (specifically IL18) and cause inflammatory ALI [79]. Specifically, a rodent model of gut ischemia/reperfusion induces a rapid increase in systemic IL-18, peaking at $1 \mathrm{~h}$ after reperfusion, paralleled by similar inflammation in lung tissue. The pulmonary inflammation associated with gut ischemia/reperfusion can be augmented by the exogenous administration of IL-18 [79]. This research is all the more intriguing when one considers that urinary IL-18 has been shown to be an early diagnostic marker for AKI (and mortality) in patients with ARDS [12] and after cardiac surgery [6]; although further investigation is warranted, given that a follow-up prospective single center trial failed to replicate this finding in adult cardiac surgery [80].

\section{Prevention/Management}

There are no definitive therapeutic trials for the treatment of PPV- and PEEP-induced renal hypoperfusion and AKI. Several small trials have shown that fluid administration and the use of vasoactive drugs (dopamine at $5 \mu \mathrm{g} / \mathrm{kg} / \mathrm{min}$ or fenoldopam) may improve renal function $[44,81,82]$, but results are not consistently positive (for example, there was no benefit of dopamine at $2 \mu \mathrm{g} /$ $\mathrm{kg} / \mathrm{min}$ ) [83]. It is unlikely that approaches focused solely on the hemodynamic and neurohormonal mechanisms of ALI-induced AKI will be clinically effective in preventing or treating this multifactorial problem, and the development of optimal interventional strategies will require more research into the molecular and cellular mechanisms involved [84].

Similarly, limited human studies suggest that ALI and PPV play a major role in AKI development. In the ARDSNet tidal volume trial, the low tidal volume group had improved survival and ventilator-free days, along with more days without circulatory, coagulation and renal failure (renal: $20 \pm 11$ vs. $18 \pm 11$ days, $\mathrm{p}=0.005$ ) [10]. Furthermore, Ranieri et al. showed in a randomized controlled trial of 44 patients with ARDS that a lung-protective mechanical ventilation strategy (lower tidal volumes and higher PEEP) induced a lesser systemic and intrapulmonary inflammatory response (as measured by serum and BAL fluid levels of TNF- $\alpha$, IL- 6 and IL-8) than the standard of care ventilation [53]. Additionally, they were able to show that this same lung-protective strategy led to fewer patients with organ system failure, with a greater decrease in the incidence of renal failure $(p<0.04)$ than any other organ dysfunction [54]. The precise pathways and mediators responsible for ventilator-induced kidney injury and other organs have not been elucidated.

\section{Clinical Effects of Permissive Hypercapnia}

Permissive hypercapnia is a commonly accepted mechanical ventilation practice, in which tidal volumes and alveolar ventilation are reduced in order to decrease ventilator-induced lung injury while treating ALI. Some of the first human work in this area by Hickling et al. [85] demonstrated that permissive hypercapnia (mean maximum $\mathrm{pCO}_{2}=62 \mathrm{~mm} \mathrm{Hg}$ ) led to a decrease in hospital mortality. In a prospective observational trial in 50 subjects with ARDS (mean $\mathrm{PaO}_{2} / \mathrm{FiO}_{2}=94$ ), they limited positive inspiratory pressure by reducing tidal volumes, allowing for spontaneous respiration and disregarded hypercapnia, and found that mortality was significantly lowered than would be predicted by APACHE II scores (16 vs. $\left.39.6 \%, p<0.001, \chi^{2}=11.64\right)$. It is this trial which laid the groundwork for the original ARDSNet trial [85]. Although it has been assumed that the beneficial effects of this lung-protective ventilatory strategy are the result of decreased stretch, lower pressure, and less mechanical trauma to injured lung, some emerging data suggest an independent lung-protective effect of permissive hypercapnia. While there are no universally accepted guidelines for the approach to mechanical ventilation in patients with ALI/ARDS, many physicians use the approach that was successful in the original ARDS Network trial of lower tidal volume ventilation [86]. This approach requires use of a tidal volume of $6 \mathrm{ml} / \mathrm{kg}$ of ideal body weight, and aims to keep static/plateau airway pressure $\leq 30 \mathrm{~cm} \mathrm{H}_{2} \mathrm{O}$, requiring permissive hypercapnia as needed to avoid ventilator-induced lung injury. Yet despite the use of this approach in clinical practice and in subsequent ARDSNet trials, the approach to permissive hypercapnia and associated acidosis remains an area of clinical uncertainty (compounded by the complex animal experimental literature discussed above) and practice variation.

Intensivists, consulting nephrologists, and other practitioners commonly grapple with questions such as: 'How much acidosis is too much acidosis?', and 'When should I consider buffering with bicarbonate or renal replacement therapy?'. The original ARDSNet low tidal volume trial protocol included arterial $\mathrm{pH}$ goals and suggested approaches to the management of acidemia and alkalemia as follows, with an arterial $\mathrm{pH}$ goal: $7.30 \leq \mathrm{pH} \leq 7.45$ : 
(a) Management of alkalemia $(\mathrm{pH}>7.45)$ : decrease ventilator rate, if possible.

(b) Management of mild acidemia $(7.15 \leq \mathrm{pH}<7.30)$ : (1) increase ventilator rate up to a maximum of 35 or until pH > 7.30 or $\mathrm{PaCO}_{2}<25 \mathrm{~mm} \mathrm{Hg}$; (2) if ventilator rate $=35$ or $\mathrm{PaCO}_{2}<25$, then bicarbonate infusion may be given.

(c) Management of severe acidemia $(\mathrm{pH}<7.15)$ : (1) increase ventilator rate to 35 ; (2) if ventilator rate $=35$ and $\mathrm{pH}<7.15$ and bicarbonate has been considered or infused, then tidal volume may be increased by $1 \mathrm{ml} / \mathrm{kg}$ until $\mathrm{pH} \geq 7.15$ (under these conditions, target plateau pressure may be exceeded).

Unfortunately, details of the frequency and extent to which these guidelines were applied in ARDSNet trials have not been published. While the available data do not permit an analysis of any impact of the use and dose of buffer therapy on outcomes, we can infer from the original report that severe acidemia was not commonly encountered. The day-1 $\mathrm{pH}$ values for those receiving low tidal volumes were (mean $\pm \mathrm{SD}$ ) $7.38 \pm 0.08$ (with $\mathrm{PaCO}_{2}$ $40 \pm 10 \mathrm{~mm} \mathrm{Hg}$ ), while those for the normal tidal volume group were $7.41 \pm 0.07$ (with $\mathrm{PaCO}_{2} 35 \pm 8 \mathrm{~mm} \mathrm{Hg}$ ). With the $\mathrm{pH}$ rising over the course of the next 7 days, there was not an apparent need to use bicarbonate therapy frequently during the course of this trial. Nonetheless, the approach to $\mathrm{pH}$ management used in this trial seems sensible, and was a component of a protocol associated with improved outcomes. We suggest that this approach should be used in clinical practice pending further research in this area. Of course, this approach was applied in patients with ALI, not those with exacerbations of chronically hypercapnic pulmonary diseases such as chronic obstructive pulmonary disease. Many such patients have chronic respiratory acidosis with compensatory metabolic alkalosis; bicarbonate should probably be used if necessary to maintain their plasma bicarbonate concentration at their chronic, pre-exacerbation level (if known), in order to facilitate liberation from mechanical ventilation when the superimposed acute respiratory failure has resolved. Finally, emerging data continue to disclose the complex effects of mechanical ventilation with permissive hypercapnia in ALI. For example, Hotchkiss et al. [87] demonstrated that increased respiratory rate can lead to a worsening of markers of lung damage (perivascular hemorrhage, and increased lung mass as a surrogate for edema). Utilizing isolated, perfused rabbit lungs they demonstrated that increased respiratory rate, in the setting of stable peak airway pressures, vascular flow and pulmonary artery pres-

Mechanical Ventilation and the Kidney sures, lead a 4.5-fold increase in lung mass as well as a 3 -fold increase in the incidence of pulmonary hemorrhage. Such data further complicate management decisions, since increased respiratory rate leading to increased pulmonary injury, and decreased rates may lead to hypoxia and hypercapnia. However, until proven otherwise by outcomes from comparable trials, we suggest that the ARDSNet protocol for mechanical ventilation in ALI is the best supported by clinical trial literature.

Permissive hypercapnia has a variety of clinical effects (in addition to the experimental phenomena discussed above), some harmful, others beneficial. In both animal models and human studies, modest hypercapnia has been shown to have reproducible hemodynamic effects that are well tolerated and reversible [88]. In both canine and human studies, these effects have included a decrease in systemic vascular resistance (systemic vasodilation), and increased cardiac output despite a decrease in cardiac contractility [89-91], although these findings are not consistent in all studies [92]. One human study evaluated the effects of buffer therapy on hypercapnic acidosis. Weber et al. [91] investigated the use of tromethamine, a buffer that does not generate $\mathrm{CO}_{2}$ (unlike bicarbonate) for this purpose. In this human study, they found that tromethamine raised cardiac contractility and mean arterial pressure (MAP). Hypercapnia also raises pulmonary vascular resistance, which is potentially harmful in patients with right ventricular dysfunction, including those with pulmonary hypertension caused by ARDS $[93,94]$. Hypercapnic pulmonary vasoconstriction may also have unfavorable effects on ventilation-perfusion matching, and result in increased intrapulmonary shunt with worsening oxygenation in ARDS [93]. On the other hand, hypercapnic acidosis also causes a rightward shift of the oxygenhemoglobin dissociation curve and improves tissue oxygen delivery in shock. Thus, although hypercapnic acidosis may cause or aggravate myocardial depression in critically ill patients, it is generally well tolerated, and buffer therapy should probably be reserved for patients with severe acidemia $(\mathrm{pH}<7.15)$. Particular caution should be exercised in the use of permissive hypercapnia in patients with pulmonary hypertension and right heart failure, in whom associated systemic vasodilation may have significant adverse hemodynamic effects. Finally, it must be remembered that the use of permissive hypercapnia is contraindicated in patients with raised intracranial pressure (cerebral edema, etc.) because of associated cerebral vasodilation and increased intracranial pressure.

What are the beneficial clinical effects of hypercapnic acidosis? Recent analysis of the ARDSNet tidal volume 
study database suggested an intrinsic benefit from hypercapnia itself, independent of the reduction of minute ventilation and airway pressures [95]. Kregenow et al. [95] showed that the adjusted odds ratio for 28-day mortality rate associated with hypercapnic acidosis (defined as $\mathrm{pH}$ $<7.35$ and $\mathrm{PaCO}_{2}>45 \mathrm{~mm} \mathrm{Hg}$ ) was 0.14 (95\% CI 0.03$0.070 ; p=0.016)$ for those receiving high tidal volumes (12 $\mathrm{ml} / \mathrm{kg}$; the injurious ventilation strategy), after controlling for severity of lung injury and comorbidities. Those who received the lung-protective strategy $(6 \mathrm{ml} /$ $\mathrm{kg})$ had an adjusted ratio of $1.18(\mathrm{p}=\mathrm{NS})$, suggesting that hypercapnic acidosis was not of additional benefit in those ventilated with lower, lung-protective tidal volumes. The observed decrease in mortality for those with respiratory acidosis in the high tidal volume arm of the trial is consistent with other emerging data suggesting an independent protective effect of hypercapnic acidosis in the setting of ALI $[61,63,64]$, and in experimental ischemia-reperfusion injury and shock [60, 62, 94]. As discussed above, several studies have shown that hypercapnic acidosis attenuates experimental ALI and ventilatorinduced lung injury $[61,63]$.

In summary, through a variety of mechanisms, permissive hypercapnia may favorably influence the course of ALI, associated ventilator-induced lung injury, and harmful organ cross talk between the injured lung and other organs, leading to a protective effect against the development of VIKI. Of course, these experimental data raise obvious concerns regarding potentially deleterious effects of the common practice of using sodium bicarbonate infusions or bicarbonate-buffered RRT to provide compensation for respiratory acidosis in mechanicallyventilated patients with ALI. However, since there is no definitive clinical confirmation of the putative independent protective effects of hypercapnic acidosis in ALI patients ventilated with low tidal volumes, the current approach to acid-base management of these complex patients using buffer therapy when severe acidosis develops seems prudent. We suggest that the guideline for $\mathrm{pH}$ management used in the ARDSNet low tidal volume trial is a sensible approach.

\section{Effects of Fluid Management and RRT on Pulmonary} Function and ALI

Fluid balance impacts pulmonary edemagenesis and oxygenation in patients with cardiac dysfunction, ALI, renal failure or other causes of pulmonary edema. Retrospective studies have shown that a negative fluid balance in patients with ALI and pulmonary capillary leak increased the number of ventilator-free days $[96,97]$. It is believed that decreased pulmonary capillary hydrostatic pressure and alveolar fluid flux leads to improved patient oxygenation and lung compliance [98]. Prospective data from a randomized controlled trial of 101 subjects found shorter duration of mechanical ventilation and ICU stay, in patients with ALI managed with negative fluid balance titrated according to extravascular lung water measurements [99]. More recently, Mangialardi et al. [100] found that hypoproteinemia and decreased oncotic pressures worsened patient outcomes in the setting of ARDS. They subsequently demonstrated that the use of a loop diuretic to achieve negative fluid balance and improve oxygenation is more effective with the concomitant use of intravenous albumin; this combination increases total plasma protein levels and oncotic pressure more than the diuretic alone $[101,102]$. However, neither of these prospective, randomized, double-blind, placebo-controlled trials were adequately powered ( $\mathrm{n}=37$ and 40$)$ to detect an effect on patient mortality, nor were they able to show a significant change in ventilator-free days.

The FACTT (Fluids and Catheters Treatment Trial) study used a $2 \times 2$ factorial design to combine two trials in 1,000 patients with ALI receiving low tidal volume mechanical ventilation. The second of these trials compared the effects of a fluid-conservative versus fluid-liberal algorithm [11]. This study was performed based on the above data, which supported the premise that negative fluid balance (fluid restriction and diuretic use) was associated with shorter ICU stays as a result of decreased length of mechanical ventilation. The primary endpoint was 60-day survival comparing a fluid liberal strategy (target central venous pressure, CVP, $<10 \mathrm{~mm} \mathrm{Hg}$ or pulmonary capillary wedge pressure, $\mathrm{PCWP},<14 \mathrm{~mm} \mathrm{Hg}$ ) versus a fluid conservative arm $(\mathrm{CVP}<4 \mathrm{~mm} \mathrm{Hg}, \mathrm{PCWP}$ $<8 \mathrm{~mm} \mathrm{Hg}$ ). Chronic kidney disease patients were not excluded (except those receiving chronic dialysis); baseline serum creatinine values in the groups were $(\mathrm{mg} / \mathrm{dl}$; mean \pm SE) fluid conservative: $1.24 \pm 0.004$; fluid liberal: $1.29 \pm 0.04 ; \mathrm{p}=0.39$ ) [11]

Not surprisingly, the fluid conservative arm received more furosemide and had a negative fluid balance over the first 7 study days $(-136 \pm 491 \mathrm{ml})$ compared with the substantial positive fluid balance of those in the liberal $\operatorname{arm}(6,992 \pm 502 \mathrm{ml} ; \mathrm{p}<0.001)$. Additionally, the fluidconservative arm had significantly more ICU-free days within the 1st week and month of the study, as well as an increased number of ventilator-free days ( $14.6 \pm 0.5$ conservative vs. $12.1 \pm 0.5$ liberal; $\mathrm{p}<0.001)$. Importantly, this underpowered study was not able to demonstrate any difference in 60 -day mortality $(25.5 \pm 1.9 \%$ conserva- 

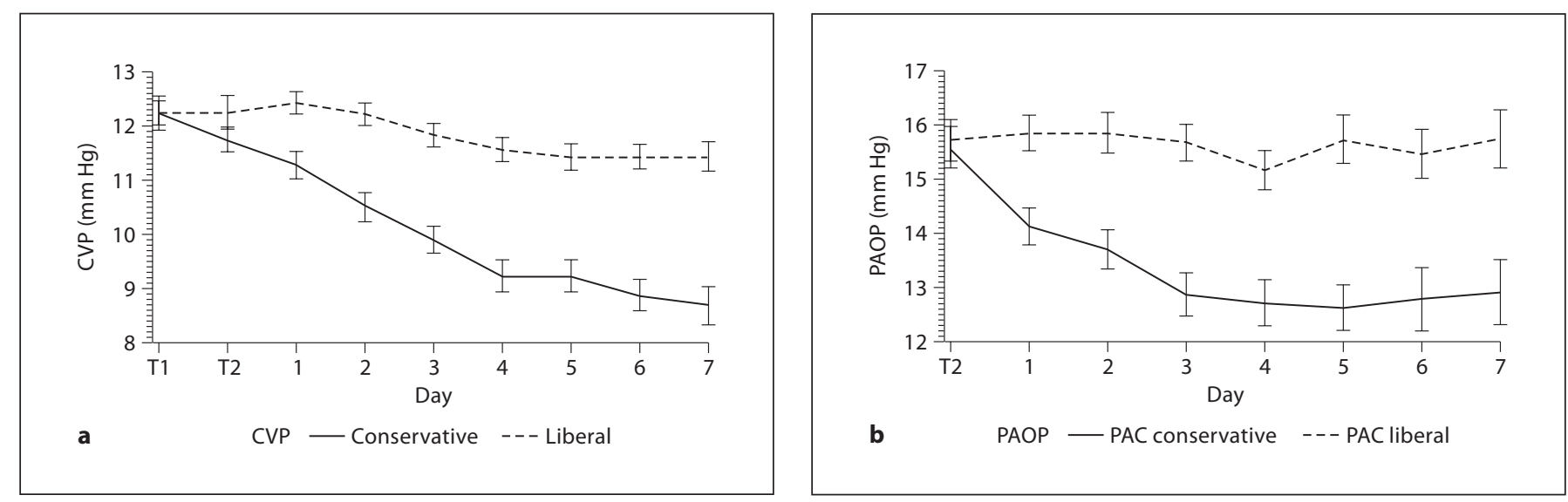

Fig. 1. Intravascular pressure over time for the two groups of the catheter portion of the FACTT trial. a For those subjects with a CVC, subjects in the fluid-liberal arm began with a CVP just above $12 \mathrm{~mm} \mathrm{Hg}$ and at day 7 they continued to maintain a CVP of $12 \mathrm{~mm} \mathrm{Hg}$. This is compared to the fluid-conservative arm who also started with a CVP of $12 \mathrm{~mm} \mathrm{Hg}$ but at day 7 had a CVP of $9 \mathrm{~mm} \mathrm{Hg}$. b We see a similar effect in the PAC group where the fluid-liberal arm had a starting PAOP of roughly $16 \mathrm{~mm} \mathrm{Hg}$ and concluded day 7 with a PAOP of $16 \mathrm{~mm}$ $\mathrm{Hg}$, while the fluid-conservative arm started at $16 \mathrm{~mm} \mathrm{Hg}$ and finished with a PAOP of $13 \mathrm{~mm} \mathrm{Hg}$. The National Heart, Lung, and Blood Institute Acute Respiratory Distress Syndrome Clinical Trials Network.

tive; $28.4 \pm 2.0 \%$ liberal; $\mathrm{p}=0.3$ ). Additionally, there was no difference in the occurrence of AKI (defined rather crudely as a serum creatinine $>2.0 \mathrm{mg} / \mathrm{dl}$ ) within the first 7 or 28 days of the study. There was, however, significantly more cardiovascular failure in the fluid-conservative group during the first 7 days of study enrollment $(\mathrm{p}=0.04)$, and more central nervous system failure in this group during days $1-7$ as well as $1-28(\mathrm{p}=0.02$ and 0.03 , respectively) [11].

Additionally, it should be noted that the other arms of the FACTT trial sought to determine the impact of fluid management guided by hemodynamic monitoring with pulmonary artery catheters (PACs) versus central venous catheters (CVCs). This trial demonstrated no benefit on survival or organ function from fluid management using the more invasive PACs [103]. However, the major positive finding of this trial was the improvement in ventilator-free survival with fluid-conservative management, whether using CVC or PAC monitoring. It is important to appreciate that this benefit was achieved by the prevention of positive fluid balance rather than the intended approach of achieving negative fluid balance. Specifically, although the conservative fluid arm protocol targeted a CVP of $4 \mathrm{~mm} \mathrm{Hg}$ or a pulmonary artery occlusion pressure (PAOP) of $8 \mathrm{~mm} \mathrm{Hg}$ compared to a CVP of 10 $14 \mathrm{~mm} \mathrm{Hg}$ or PAOP of $14-18 \mathrm{~mm} \mathrm{Hg}$ in the fluid-liberal arm, the fluid-conservative targets were not generally achieved. Subjects with CVC began the trial with an average CVP of 12-13 mm Hg; those in the fluid-liberal arm only decreased slightly to $11-12 \mathrm{~mm} \mathrm{Hg}$ over the first 7 days, while those in the fluid conservative decreased to 8-9 mm Hg (fig. 1a). In the PAC arm, both groups started with a PAOP of $15-16 \mathrm{~mm} \mathrm{Hg}$, with those in the fluidliberal arm finishing close to $16 \mathrm{~mm} \mathrm{Hg}$, which was higher than the 12-14 mm Hg for the fluid-conservative group (fig. 1b). Regardless of the monitoring catheter in place, while the fluid-conservative arms clearly achieved tighter fluid control, analysis of their intravascular fluid pressures demonstrates that the fluid-conservative group did not achieve the low level of filling pressure targeted by the study protocol, and this may have contributed to the lack of a significant difference in the overall mortality and in the AKI rates between the fluid-liberal and conservative arms. Examination of the cumulative fluid balance data underscores this impression. Figure 2 demonstrates the cumulative fluid balance in the fluid-conservative and fluid-liberal arms of this trial, along with two patient populations which were recently studied in the setting of ALI. The first of these prior ARDSNet trials was the ALVEOLI study [104] which tested lower tidal volumes with higher PEEP and, after enrolling 549 patients, found no further improvement in survival with higher PEEP. The other group in this figure is those patients who received the lung-protective $6 \mathrm{ml} / \mathrm{kg}$ tidal volumes in the original ARDSNet study (ARMA - $6 \mathrm{ml}$ ) [10]. Of note, these data suggest that the fluid-liberal arm accurately serves as a 


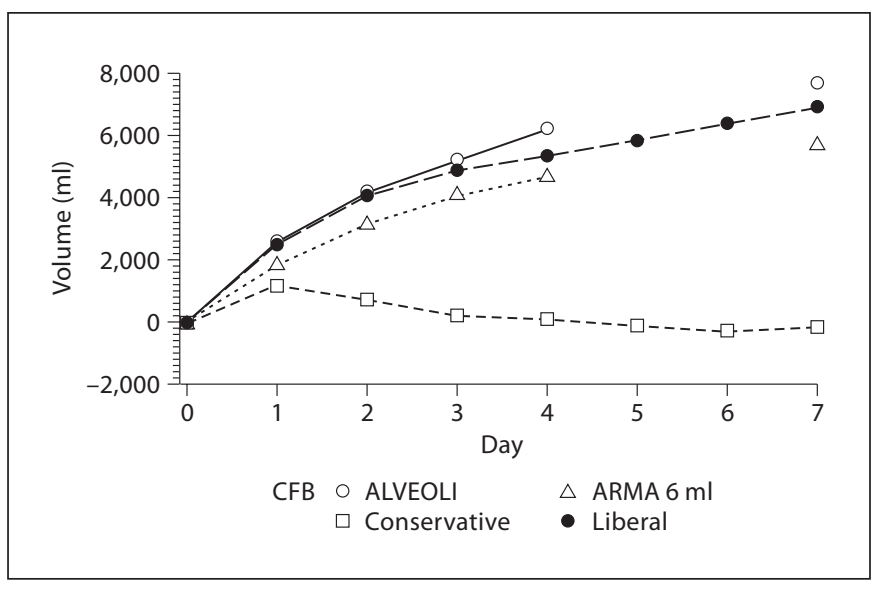

Fig. 2. Net fluid balance for fluid liberal and fluid conservative arms of the FACTT trial as well as for the ALVEOLI trial, and the $6 \mathrm{ml} / \mathrm{kg}$ tidal volume of the original ARDSNet trial. The National Heart, Lung, and Blood Institute Acute Respiratory Distress Syndrome Clinical Trials Network.

'real-world' example of fluid balance in ALI patients, as the net fluid in (roughly 5.61 positive over the first 4 days and 7.11 positive for the first 7 days) is similar to the balance achieved in previous ARDSNet trials (which did not attempt to control fluid status). Conversely, the fluidconservative arm was not able to obtain a net negative fluid balance over the first 4 days, but rather a net even fluid balance ( 0.21 net positive in 4 days; with 0.011 positive on day 7). As such, those clinicians looking to replicate the protocols set forth in this landmark trial in clinical practice need only to ensure that they achieve a net even fluid balance, rather than needing to achieve negative fluid balance. Similarly, one must recognize that this trial had a very intricate protocol to account for hemodynamic instability. At any sign of shock (defined as a MAP of less than $60 \mathrm{~mm} \mathrm{Hg}$, end-organ hypoperfusion), the protocol required the discontinuation of diuretics, as well as fluid boluses and the initiation and/or escalation of vasoactive medications (dobutamine, norepinephrine, epinephrine, vasopressin, or phenylephrine) as needed. This last point is yet another important caveat to consider when attempting to treat patients with ALI in a manner similar to the fluid-conservative arm of the FACTT trial.

In summary, although in the FACTT study improved pulmonary function and lung mechanics did not translate into improved 60-day patient survival (the primary trial endpoint), improved lung function did impact the more restricted endpoint of ventilator-free survival. As discussed above, the failure to achieve actual negative fluid balance may have led to a less than maximal improvement in 60-day survival. Alternatively, unquantified adverse effects of fluid restriction and diuretics on non-pulmonary organs may have led to competing morbidities in the dry group. For example, although not statistically significant, there was a trend towards higher serum creatinine levels in the conservative group $(\mathrm{p}=0.07)$, along with significantly higher blood urea nitrogen and bicarbonate levels. On the other hand, as previously noted there was a trend towards an increased need for RRT in the fluid-liberal group (14\%) compared to the fluidconservative group $(10 \% ; p=0.06)$. However, it is important to note that the need for RRT is a subjective endpoint that is open to bias due to the unblinded nature of the trial. Elevated serum creatinine and increased blood urea nitrogen were perhaps tolerated in the fluid-conservative arm, due to the trial's aim to run the subjects at lower intravascular volumes (CVP of $4 \mathrm{~mm} \mathrm{Hg}$ and PCWP of 8 $\mathrm{mm} \mathrm{Hg}$ ). Conversely, it is reasonable to suppose that positive fluid balance would be tolerated to a greater extent in the fluid-liberal group, but that eventually excess volume might lead to an increased rate of RRT. Further investigation of the role of fluid management, in an appropriately powered study, in the setting of ALI would be better served by the use of more precise definitions and biomarkers of AKI, among other measures of adequate tissue perfusion.

As discussed, although the fluid-conservative strategy did not improve all-cause mortality in ALI, the number of ventilator-free days alive was significantly increased, and this has led by extrapolation (appropriate or otherwise) to an increased interest in the use of RRT to control fluid balance in patients with ALI and an inadequate response to fluid restriction and diuretics [11]. It is should be noted that renal failure requiring dialysis was an exclusion criterion in the FACTT, and the fluid management algorithm and trial protocol participation were suspended if dialysis-requiring AKI developed following enrollment. However, the concept that fluid overload contributes to adverse patient outcomes in AKI is supported by the work of Goldstein et al. [105], who have determined the impact of fluid overload in the setting of pediatric AKI (in the pediatric ICU (PICU) and in stem cell transplants). In a prospective observational study of 21 PICU patients receiving continuous RRT (CRRT), Goldstein et al. [105] demonstrated that the degree of fluid overload [defined as ((fluid in - fluid out)/PICU admission weight) $\cdot 100]$ at continuous veno-venous hemo- 
diafiltration (CVVHDF) initiation was significantly lower $(p=0.03)$ in survivors (16.4 $\pm 13.8 \%$; range: $2-44.8 \%)$ compared with nonsurvivors $(34 \pm 21 \%$; range: $7.5-$ $80 \%)$. This finding was supported by recent analysis of pediatric stem cell transplant patients who develop AKI. With a protocol in place to initiate CRRT in those subjects who develop $10 \%$ fluid overload, Michael et al. [106] showed that all survivors $(n=11$, of a total of 26 subjects who had stem cell transplant and AKI) were able to maintain a $<10 \%$ fluid overload status. This included 4 subjects who required CRRT to maintain appropriate fluid balance. They concluded that while not definitive, the ability to maintain euvolemia ( $<10 \%$ fluid overload) is critical for pediatric patients with AKI in the setting of stem cell transplantation [106]. These findings are consistent with those of Payen and the Sepsis Occurrence in Acutely Ill Patients (SOAP) Investigators. This group showed that in a 3,147 adult multicenter European observational study in patients with AKI (crudely defined as serum creatinine $>3.5 \mathrm{mg} / \mathrm{dl}$ or urine output less than $500 \mathrm{ml} /$ day), mean daily fluid balance was significantly more positive amongst nonsurvivors than among survivors [(1/day) $0.98 \pm 1.5$ vs. $0.15 \pm 1.06$; $p<0.001$ ] [107]. Of course, all of these data documenting the association of positive fluid balance in AKI with adverse outcomes do not prove that interventions to control fluid accumulation would accordingly improve survival. Nonetheless, while there have been no definitive, randomized trials to determine the effects of fluid balance control with RRT in the setting of ALI or AKI, it is clear that volume overload remains an important determinant in adverse patient outcomes and additional studies are needed to guide practice in these common clinical scenarios.

Meanwhile, the correct approach to the use of RRT to control azotemia is increasingly guided by randomized, controlled dosing trials [108-111]. However, the correct approach to use of RRT to control fluid balance or buffer permissive hypercapnic respiratory acidosis is not guided by high-level evidence. As discussed above, emerging experimental evidence suggests that respiratory acidosis may have protective effects in ALI, and buffering with bicarbonate may be harmful, but such correction is commonly made with CRRT in patients with combined ALI and AKI $[62,63,95]$. Of course, support with RRT has many other potentially beneficial effects with patients with combined AKI and ALI. By extrapolation from the literature describing beneficial effects of negative fluid balance in ALI, the use of intermittent or continuous renal replacement therapies to control fluid balance should have benefit in this patient population. However, there is a lack of clinical trial data to support this assumption. Most trials of RRT to treat patients with ALI have focused on approaches to treat inflammation rather than achieve negative fluid balance.

Continuous hemofiltration with a negative or zero net fluid balance has a hypothesized therapeutic benefit in patient with ALI due to its ability to remove inflammatory and humoral mediators of AKI, ALI, and sepsis from the circulation. Additionally, patients could derive some therapeutic benefit (improved oxygenation/gas exchange) from fluid removal resulting in decreased pulmonary vascular congestion (lower cardiac filling pressures) if the prescribed therapy maintains even fluid balance (i.e. prevents positive fluid balance) or achieves negative fluid balance. Several animal models (porcine and canine) have reported success in reducing pulmonary edema via these aforementioned mechanisms [112-115]. The models employed in these studies have varied but include oleic acidinduced ALI [113], endotoxin-induced ALI [112, 114] and experimental models of pancreatitis [115]. Some of these studies have documented success both clinically as well as via reduced plasma levels of a variety of inflammatory markers, including IL- 6 , NF- $\kappa$ B, and others $[113,115]$.

Unfortunately, human trials using modern CRRT have yielded mixed results. Several studies performed in the 1980s and 1990s had conflicting results regarding gas exchange and outcomes of patients with ALI receiving RRT [116], but some more recent trials have been encouraging. Recent studies investigating the role of RRT specifically in ARDS have also had mixed results, in part due to study design limitations. Piccinni et al. [117] retrospectively reviewed 80 cases of septic shock associated with ARDS. They compared 40 individuals who received early isovolemic hemofiltration with 40 individuals who received conventional therapy. They found that hemofiltration improved oxygenation (as measured by $\mathrm{PaO}_{2} / \mathrm{FiO}_{2}$; $\mathrm{p}<0.05$ ) and hemodynamic status (as measured by MAP and pressor dose; $\mathrm{p}<0.05$ ). Additionally, the subjects receiving the early hemofiltration had shorter ICU stays $(\mathrm{p}<0.002)$ and improved 28 -day survival (55 vs. $27.5 \%$, $\mathrm{p}<0.05)$. Some of these findings were supported by a smaller uncontrolled, prospective study of 10 children with ARDS associated with cancer therapy. In this trial only 4 of the 10 children had AKI, but all underwent early CVVHDF to achieve a negative or zero fluid balance. Ninety percent of the subjects achieved resolution of their ARDS; with 8 of the 10 patients alive at the 18 -month follow-up [118]. On the other hand, Hoste et al. [116] conducted a retrospective study of 37 patients with AKI and ALI, all of whom underwent CVVHDF. They found no 
benefit when comparing hemodynamics (MAP, CVP, etc.), mechanical ventilation requirements $\left(\mathrm{FiO}_{2}, \mathrm{PEEP}\right.$, etc.) or gas exchange $\left(\mathrm{PaO}_{2}, \mathrm{PaCO}_{2}\right.$, etc.) parameters from $24 \mathrm{~h}$ before RRT initiation with those from the first $24 \mathrm{~h}$ after initiation. It is clear that additional prospective trials are required to determine the optimal approach to delivery of RRT in the treatment of patients with AKI and ALI.

\section{Conclusion}

In conclusion, it is evident that there is a deleterious bidirectional relationship between the AKI and ALI. Increasing evidence points to cross talk between these two distant organs, and shows that injury to one organ may initiate and aggravate injury to the other. Recent data show that the kidneys play an important role in the production and elimination of mediators of inflammation and ALI. Conversely, exposure to the inflammatory milieu of ALI and mechanical ventilation-induced injury may precipitate the onset of AKI, and we suggest that this phenomenon might be termed ventilator-induced kidney injury (VIKI). While there have been recent advances in approaches to limit ventilator-induced lung injury and decrease the duration of mechanical ventilatory support, the net effect of these advances on the incidence and severity of AKI in critically ill patients remains to be determined. Close collaboration between intensivists and nephrologists is required to optimize management and improve outcomes of patients with ALI and AKI.

\section{References}

$\checkmark 1$ Bellomo R, et al: Acute renal failure - definition, outcome measures, animal models, fluid therapy and information technology needs: the Second International Consensus Conference of the Acute Dialysis Quality Initiative (ADQI) Group. Crit Care 2004;8: R204-R212.

$\checkmark 2$ Mehta RL, et al: Acute Kidney Injury Network: report of an initiative to improve outcomes in acute kidney injury. Crit Care 2007; 11:R31.

3 Han WK, et al: Urinary biomarkers in the early diagnosis of acute kidney injury. Kidney Int 2007;5:5.

4 Koyner JL, et al: Urinary cystatin C as an early biomarker of acute kidney injury following adult cardiothoracic surgery. Kidney Int 2008;23:23

5 Mishra J, et al: Neutrophil gelatinase-associated lipocalin (NGAL) as a biomarker for acute renal injury after cardiac surgery. Lancet 2005;365:1231-1238.

6 Parikh CR, et al: Urinary IL-18 is an early predictive biomarker of acute kidney injury after cardiac surgery. Kidney Int 2006;70: 199-203.

$>7$ Hall J, et al (eds): Principles of Critical Care, ed 2. New York, McGraw-Hill, vol 1, 1992, pp 19-21.

8 Ware LB, et al: Clinical practice. Acute pulmonary edema. N Engl J Med 2005;353: 2788-2796.

9 Bernard GR, et al: The American-European Consensus Conference on ARDS. Definitions, mechanisms, relevant outcomes, and clinical trial coordination. Am J Respir Crit Care Med 1994;149:818-824.
10 ARDSNet: Ventilation with lower tidal volumes as compared with traditional tidal volumes for acute lung injury and the acute respiratory distress syndrome. The Acute Respiratory Distress Syndrome Network. N Engl J Med 2000;342:1301-1308.

-11 Wiedemann HP, et al: Comparison of two fluid-management strategies in acute lung injury. N Engl J Med 2006;354:2564-2575.

12 Parikh CR, et al: Urine IL-18 is an early diagnostic marker for acute kidney injury and predicts mortality in the intensive care unit. J Am Soc Nephrol 2005; 16:3046-3052.

13 Ketoconazole for early treatment of acute lung injury and acute respiratory distress syndrome: a randomized controlled trial. The ARDS Network. JAMA 2000;283:19952002.

14 Randomized, placebo-controlled trial of lisofylline for early treatment of acute lung injury and acute respiratory distress syndrome. Crit Care Med 2002;30:1-6.

15 Liu KD, et al: Predictive and pathogenetic value of plasma biomarkers for acute kidney injury in patients with acute lung injury. Crit Care Med 2007;23:23.

16 Rubenfeld GD, et al: Incidence and outcomes of acute lung injury. N Engl J Med 2005;353: 1685-1693.

17 Bersten AD, et al: Incidence and mortality of acute lung injury and the acute respiratory distress syndrome in three Australian States. Am J Respir Crit Care Med 2002;165:443448.

$>18$ Brun-Buisson C, et al: Epidemiology and outcome of acute lung injury in European intensive care units. Results from the ALIVE study. Intensive Care Med 2004;30:51-61.
$>19$ Hughes M, et al: Acute respiratory distress syndrome: an audit of incidence and outcome in Scottish intensive care units. Anaesthesia $2003 ; 58: 838-845$

20 Liu KD, et al: Advances in critical care for the nephrologist: acute lung injury/ARDS. Clin J Am Soc Nephrol 2008;3:578-586.

21 Meyer NJ, Garcia JG: Wading into the genomic pool to unravel acute lung injury genetics. Proc Am Thorac Soc 2007;4:69-76.

22 Chertow G, et al: Independent association between acute renal failure and mortality following cardiac surgery. Am J Med 1998; 104:343-348.

23 Uchino S, et al: Acute renal failure in critically ill patients: a multinational, multicenter study. JAMA 2005;294:813-818.

$>24$ Star RA: Treatment of acute renal failure. Kidney Int 1998;54:1817-1831.

-25 Lassnigg A, et al: Minimal changes of serum creatinine predict prognosis in patients after cardiothoracic surgery: a prospective cohort study. J Am Soc Nephrol 2004;15:15971605

26 Murray P, et al: Renal effect of critical illness; in Murray P, Brady B, Hall R (eds): Intensive Care Unit Nephrology. London, Taylor \& Francis, 2005, pp 47-67.

27 Drury DR, et al: The effects of continuous pressure breathing on kidney function. J Clin Invest 1947;26:945-951.

-28 Pannu N, et al: Effect of mechanical ventilation on the kidney. Best Pract Res Clin Anaesthesiol 2004;18:189-203.

29 Annat G, et al: Effect of PEEP ventilation on renal function, plasma renin, aldosterone, neurophysins and urinary $\mathrm{ADH}$, and prostaglandins. Anesthesiology 1983;58:136-141. 
-30 Priebe HJ, et al: Mechanisms of renal dysfunction during positive end-expiratory pressure ventilation. J Appl Physiol 1981;50: 643-649.

31 Mullins RJ, et al: Mechanisms of impaired renal function with PEEP. J Surg Res 1984;37: 189-196.

32 Kaukinen S, et al: Positive end expiratory pressure ventilation, renal function and renin. Ann Clin Res 1979;11:58-62.

33 Qvist J, et al: Hemodynamic responses to mechanical ventilation with PEEP: the effect of hypervolemia. Anesthesiology 1975;42: 45-55.

34 Doty JM, et al: Effects of increased renal parenchymal pressure on renal function. J Trauma 2000;48:874-877.

- 35 Sugerman HJ: Effects of increased intra-abdominal pressure in severe obesity. Surg Clin North Am 2001;81:1063-1075.

-36 Shear W, et al: Acute kidney dysfunction secondary to the abdominal compartment syndrome. J Nephrol 2006;19:556-565.

37 Moore ES, et al: Effects of positive pressure ventilation on intrarenal blood flow in infant primates. Pediatr Res 1974;8:792-796.

38 Hall SV, et al: Renal hemodynamics and function with continuous positive-pressure ventilation in dogs. Anesthesiology 1974;41: 452-461.

39 Gauer OH, et al: The effect of negative pressure breathing on urine flow. J Clin Invest 1954;33:287-296.

-40 Fewell JE, et al: Renal denervation eliminates the renal response to continuous positivepressure ventilation. Proc Soc Exp Biol Med 1979;161:574-578.

-41 Bark H, et al: Elevations in plasma ADH levels during PEEP ventilation in the dog: mechanisms involved. Am J Physiol 1980; 239:E474-E481.

42 Andrivet $\mathrm{P}$, et al: Hormonal interactions and renal function during mechanical ventilation and ANF infusion in humans. J Appl Physiol 1991;70:287-292.

-43 Farge D, et al: Interactions between hemodynamic and hormonal modifications during PEEP-induced antidiuresis and antinatriuresis. Chest 1995;107:1095-1100.

-44 Ramamoorthy C, et al: Aggressive hydration during continuous positive-pressure ventilation restores atrial transmural pressure, plasma atrial natriuretic peptide concentrations, and renal function. Crit Care Med 1992;20:1014-1019.

-45 Sata T, et al: Increased release of alpha-atrial natriuretic peptide during controlled mechanical ventilation with positive end-expiratory pressure in humans. J Anesth 1988;2: 119-123.

46 Scheel PJ, et al: Uremic lung: new insights into a forgotten condition. Kidney Int 2008; 74:849-851

47 Bass HE, et al: Pulmonary changes in uremia. J Am Med Assoc 1950;144:819-823.
48 Kelly KJ: Distant effects of experimental renal ischemia/reperfusion injury. J Am Soc Nephrol 2003;14:1549-1558.

49 Vieira JM Jr, et al: Effect of acute kidney injury on weaning from mechanical ventilation in critically ill patients. Crit Care Med 2007;35:184-191.

50 Kim do J, et al: Comparison of experimental lung injury from acute renal failure with injury due to sepsis. Respiration 2006;73:815824.

51 Cohen J: The immunopathogenesis of sepsis. Nature 2002;420:885-891.

52 Imai Y, et al: Injurious mechanical ventilation and end-organ epithelial cell apoptosis and organ dysfunction in an experimental model of acute respiratory distress syndrome. JAMA 2003;289:2104-2112.

53 Ranieri VM, et al: Effect of mechanical ventilation on inflammatory mediators in patients with acute respiratory distress syndrome: a randomized controlled trial. JAMA 1999;282:54-61.

54 Ranieri VM, et al: Mechanical ventilation as a mediator of multisystem organ failure in acute respiratory distress syndrome. JAMA 2000;284:43-44.

55 Douillet CD, et al: Mechanical ventilation alters airway nucleotides and purinoceptors in lung and extrapulmonary organs. Am J Respir Cell Mol Biol 2005;32:52-58.

56 Kuiper JW, et al: Production of endothelin-1 and reduced blood flow in the rat kidney during lung-injurious mechanical ventilation. Anesth Analg 2008;107:1276-1283.

57 Hoag JB, et al: Effects of acid aspiration-induced acute lung injury on kidney function. Am J Physiol Renal Physiol 2008;294:F900F908.

58 Moncada S, et al: The L-arginine-nitric oxide pathway. N Engl J Med 1993;329:20022012.

59 Choi WI, et al: Systemic microvascular leak in an in vivo rat model of ventilator-induced lung injury. Am J Respir Crit Care Med 2003; 167:1627-1632.

60 Nomura F, et al: Effects of hypercarbic acidotic reperfusion on recovery of myocardial function after cardioplegic ischemia in neonatal lambs. Circulation 1994;90:II321II327.

61 Kavanagh BP, et al: Hypercapnia: permissive and therapeutic. Minerva Anestesiol 2006 72:567-576.

-62 Coakley RJ, et al: Ambient $\mathrm{pCO}_{2}$ modulates intracellular $\mathrm{pH}$, intracellular oxidant generation, and interleukin- 8 secretion in human neutrophils. J Leukoc Biol 2002;71: 603-610.

63 Laffey JG, et al: Therapeutic hypercapnia reduces pulmonary and systemic injury following in vivo lung reperfusion. Am J Respir Crit Care Med 2000;162:2287-2294.

64 Tak PP, et al: Inhibitor of nuclear factor kap$\mathrm{paB}$ kinase beta is a key regulator of synovial inflammation. Arthritis Rheum 2001;44: 1897-1907.
65 O'Croinin DF, et al: Sustained hypercapnic acidosis during pulmonary infection increases bacterial load and worsens lung injury. Crit Care Med 2008;36:2128-2135.

66 Chonghaile MN, et al: Hypercapnic acidosis attenuates lung injury induced by established bacterial pneumonia. Anesthesiology 2008;109:837-848.

67 Kramer AA, et al: Renal ischemia/reperfusion leads to macrophage-mediated increase in pulmonary vascular permeability. Kidney Int 1999;55:2362-2367.

68 Rabb H, et al: Acute renal failure leads to dysregulation of lung salt and water channels. Kidney Int 2003;63:600-606.

$69 \mathrm{Ma} \mathrm{T}$, et al: Lung fluid transport in aquaporin-5 knockout mice. J Clin Invest 2000;105: 93-100.

70 Hummler E, et al: Role of the epithelial sodium channel in lung liquid clearance. Chest 1997;111(suppl 6):113S

71 Hummler E, et al: Early death due to defective neonatal lung liquid clearance in alphaENaC-deficient mice. Nat Genet 1996;12: 325-328.

72 Hoke TS, et al: Acute renal failure after bilateral nephrectomy is associated with cytokine-mediated pulmonary injury. J Am Soc Nephrol 2007;18:155-164.

73 Hassoun HT, et al: Ischemic acute kidney injury induces a distant organ functional and genomic response distinguishable from bilateral nephrectomy. Am J Physiol Renal Physiol 2007;293:F30-F40.

74 Klein CL, et al: Interleukin-6 mediates lung injury following ischemic acute kidney injury or bilateral nephrectomy. Kidney Int 2008;74:901-909.

75 Dodd-o JM, et al: Interactive effects of mechanical ventilation and kidney health on lung function in an in vivo mouse model. Am J Physiol Lung Cell Mol Physiol 2008;10: 10.

76 Zarbock A, et al: Complete reversal of acidinduced acute lung injury by blocking of platelet-neutrophil aggregation. J Clin Invest 2006;116:3211-3219.

77 Deng J, et al: Alpha-melanocyte-stimulating hormone inhibits lung injury after renal ischemia/reperfusion. Am J Respir Crit Care Med 2004;169:749-756.

78 Grigoryev DN, et al: The local and systemic inflammatory transcriptome after acute kidney injury. J Am Soc Nephrol 2008;19: 547-558.

79 Yang YJ, et al: Role of interleukin 18 in acute lung inflammation induced by gut ischemia reperfusion. World J Gastroenterol 2005;11: 4524-4529.

80 Haase M, et al: Urinary interleukin-18 does not predict acute kidney injury after adult cardiac surgery: a prospective observational cohort study. Crit Care 2008;12:R96.

81 Pannu N, et al: Mechanical ventilation and renal function: an area for concern? Am J Kidney Dis 2002;39:616-624. 
82 Poinsot O, et al: Fenoldopam improves renal hemodynamics impaired by positive end-expiratory pressure. Anesthesiology 1993;79: 680-684.

$>83$ Kim YJ, et al: Does low dose dopamine attenuate the decrease of renal function in the treatment of patients under controlled mechanical ventilation with positive end expiratory pressure? Yonsei Med J 1998;39:189195.

$>84$ Kuiper JW, et al: Mechanical ventilation and acute renal failure. Crit Care Med 2005;33: 1408-1415.

85 Hickling KG, et al: Low mortality associated with low volume pressure limited ventilation with permissive hypercapnia in severe adult respiratory distress syndrome. Intensive Care Med 1990;16:372-377.

86 Ventilation with lower tidal volumes as compared with traditional tidal volumes for acute lung injury and the acute respiratory distress syndrome. The Acute Respiratory Distress Syndrome Network. N Engl J Med 2000;342:1301-1308.

87 Hotchkiss JR Jr, et al: Effects of decreased respiratory frequency on ventilator-induced lung injury. Am J Respir Crit Care Med 2000; 161:463-468.

88 Feihl F, et al: Permissive hypercapnia. How permissive should we be? Am J Respir Crit Care Med 1994;150:1722-1737.

89 Thorens JB, et al: Effects of rapid permissive hypercapnia on hemodynamics, gas exchange, and oxygen transport and consumption during mechanical ventilation for the acute respiratory distress syndrome. Intensive Care Med 1996;22:182-191.

$\checkmark 90$ Walley KR, et al: Acute respiratory acidosis decreases left ventricular contractility but increases cardiac output in dogs. Circ Res 1990;67:628-635.

-91 Weber T, et al: Tromethamine buffer modifies the depressant effect of permissive hypercapnia on myocardial contractility in patients with acute respiratory distress syndrome. Am J Respir Crit Care Med 2000;162: 1361-1365.

92 McIntyre RC Jr, et al: Cardiopulmonary effects of permissive hypercapnia in the management of adult respiratory distress syndrome. J Trauma 1994;37:433-438.

93 Hickling KG, et al: Permissive hypercapnia in ARDS and its effect on tissue oxygenation. Acta Anaesthesiol Scand Suppl 1995;107: 201-208.
-94 Rose CE Jr, et al: Right ventricular performance during increased afterload impaired by hypercapnic acidosis in conscious dogs. Circ Res 1983;52:76-84.

$\$ 95$ Kregenow DA, et al: Hypercapnic acidosis and mortality in acute lung injury. Crit Care Med 2006;34:1-7.

$\$ 96$ Alsous F, et al: Negative fluid balance predicts survival in patients with septic shock: a retrospective pilot study. Chest 2000;117: 1749-1754

$\$ 97$ Schuster DP: The case for and against fluid restriction and occlusion pressure reduction in adult respiratory distress syndrome. New Horiz 1993;1:478-488.

$\$ 98$ Lewis CA, et al: Understanding and managing fluid balance in patients with acute lung injury. Curr Opin Crit Care 2004;10: 13-17.

$\$ 99$ Mitchell JP, et al: Improved outcome based on fluid management in critically ill patients requiring pulmonary artery catheterization. Am Rev Respir Dis 1992;145: 990-998.

100 Mangialardi RJ, et al: Hypoproteinemia predicts acute respiratory distress syndrome development, weight gain, and death in patients with sepsis. Ibuprofen in Sepsis Study Group. Crit Care Med 2000;28:31373145.

101 Martin GS, et al: Albumin and furosemide therapy in hypoproteinemic patients with acute lung injury. Crit Care Med 2002;30: 2175-2182.

102 Martin GS, et al: A randomized, controlled trial of furosemide with or without albumin in hypoproteinemic patients with acute lung injury. Crit Care Med 2005;33: 1681-1687.

103 Wheeler AP, et al: Pulmonary-artery versus central venous catheter to guide treatment of acute lung injury. N Engl J Med 2006;354: 2213-2224.

104 Brower RG, et al: Higher versus lower positive end-expiratory pressures in patients with the acute respiratory distress syndrome. N Engl J Med 2004;351:327-336.

105 Goldstein SL, et al: Outcome in children receiving continuous venovenous hemofiltration. Pediatrics 2001;107:1309-1312.
106 Michael M, et al: Fluid overload and acute renal failure in pediatric stem cell transplant patients. Pediatr Nephrol 2004;19: 91-95.

107 Payen D, et al: A positive fluid balance is associated with a worse outcome in patients with acute renal failure. Crit Care 2008;12: R74.

108 Ronco C, et al: Effects of different doses in continuous veno-venous haemofiltration on outcomes of acute renal failure: a prospective randomised trial. Lancet 2000; 356:26-30.

109 Schiffl H, et al: Daily hemodialysis and the outcome of acute renal failure. $\mathrm{N}$ Engl J Med 2002;346:305-310.

110 Palevsky PM, et al: Design of the VA/NIH Acute Renal Failure Trial Network (ATN) Study: intensive versus conventional renal support in acute renal failure. Clin Trials 2005;2:423-435.

111 Palevsky PM, et al: Intensity of renal support in critically ill patients with acute kidney injury. N Engl J Med 2008;359:7-20.

112 Stein B, et al: The consequences of continuous haemofiltration on lung mechanics and extravascular lung water in a porcine endotoxic shock model. Intensive Care Med 1991;17:293-298.

113 Su X, et al: Effect of continuous hemofiltration on hemodynamics, lung inflammation and pulmonary edema in a canine model of acute lung injury. Intensive Care Med 2003; 29:2034-2042.

114 Ullrich R, et al: Continuous venovenous hemofiltration improves arterial oxygenation in endotoxin-induced lung injury in pigs. Anesthesiology 2001;95:428-436.

115 Yan XW, et al: Effects of high-volume continuous hemofiltration on experimental pancreatitis associated lung injury in pigs. Int J Artif Organs 2006;29:293-302.

$>116$ Hoste EA, et al: No early respiratory benefit with CVVHDF in patients with acute renal failure and acute lung injury. Nephrol Dial Transplant 2002;17:2153-2158.

117 Piccinni P, et al: Early isovolaemic haemofiltration in oliguric patients with septic shock. Intensive Care Med 2006;32:80-86.

118 DiCarlo JV, et al: Continuous veno-venous hemofiltration may improve survival from acute respiratory distress syndrome after bone marrow transplantation or chemotherapy. J Pediatr Hematol Oncol 2003;25: 801-805. 